\title{
Video Article \\ Non-invasive Assessment of the Efficacy of New Therapeutics for Intestinal Pathologies Using Serial Endoscopic Imaging of Live Mice
}

\author{
Matthias Ernst ${ }^{1,2,3}$, Adele Preaudet ${ }^{1}$, Tracy Putoczki ${ }^{1,2}$ \\ ${ }^{1}$ The Walter and Eliza Hall Institute for Medical Research \\ ${ }^{2}$ The Department of Medical Biology, University of Melbourne \\ ${ }^{3}$ Olivia Newton-John Cancer Research Institute
}

Correspondence to: Tracy Putoczki at putoczki.t@wehi.edu.au

URL: https://www.jove.com/video/52383

DOI: doi:10.3791/52383

Keywords: Medicine, Issue 97, cancer, colitis, colon, endoscopy, mucosa, therapy.

Date Published: 3/10/2015

Citation: Ernst, M., Preaudet, A., Putoczki, T. Non-invasive Assessment of the Efficacy of New Therapeutics for Intestinal Pathologies Using Serial Endoscopic Imaging of Live Mice. J. Vis. Exp. (97), e52383, doi:10.3791/52383 (2015).

\section{Abstract}

Animal models of inflammatory bowel disease (IBD) and colorectal cancer (CRC) have provided significant insight into the cell intrinsic and extrinsic mechanisms that contribute to the onset and progression of intestinal diseases. The identification of new molecules that promote these pathologies has led to a flurry of activity focused on the development of potential new therapies to inhibit their function. As a result, various preclinical mouse models with an intact immune system and stromal microenvironment are now heavily used. Here we describe three experimental protocols to test the efficacy of new therapeutics in pre-clinical models of (1) acute mucosal damage, (2) chronic colitis and/or colitis-associated colon cancer, and (3) sporadic colorectal cancer. We also outline procedures for serial endoscopic examination that can be used to document the therapeutic response of an individual tumor and to monitor the health of individual mice. These protocols provide complementary experimental platforms to test the effectiveness of therapeutic compounds shown to be well tolerated by mice.

\section{Video Link}

The video component of this article can be found at https://www.jove.com/video/52383/

\section{Introduction}

Colorectal cancer $(\mathrm{CRC})$ is the $4^{\text {th }}$ most common cause of malignancy worldwide ${ }^{1}$. Despite the significant progress in our understanding of the familial basis of this disease, genetic predisposition only contributes to $\sim 20 \%$ of CRC cases ${ }^{2}$. The remainder are attributed to numerous extrinsic and environmental factors, including chronic inflammation. In humans, the link between chronic inflammation and colon cancer is evident in ulcerative colitis (UC) patients, who have a greater risk of developing colitis-associated colon cancer (CAC), depending on the duration, extent and severity of the inflammatory disease ${ }^{3-5}$. Accordingly, novel therapies are in development to control the immune response and the associated production of growth promoting factors by the inflammatory tumor microenvironment ${ }^{6-8}$. There is an increasing requirement for appropriate preclinical animal models to characterize the therapeutic efficacy of these drugs against the development and progression of disease.

Mouse models have unequivocally demonstrated that the inflammatory microenvironment contributes to CRC progression, even in the absence of overt inflammation ${ }^{9,10}$. These models include the use of the polysaccharide dextran sulfate sodium (DSS), provided in the drinking water of mice, to model epithelial injury and acute and chronic inflammatory bowel disease (IBD) ${ }^{11,12}$. Although the mechanism by which DSS induces mucosal damage and colitis is not completely understood, some studies suggest that DSS inhibits cellular reverse transcriptase and ribonuclease activities within cells, or promotes the formation of nano-lipid complexes that fuse with the colonic membrane leading to epithelial damage $^{13,14}$. Modifications to the standard DSS model have also provided significant insight into the mechanisms by which colonic epithelial cells maintain tissue homeostasis and regulate mucosal immune responses ${ }^{15}$.

Intraperitoneal administration of Azoxymethane (AOM) alone, or in combination with DSS, provides a model for examining the interplay between somatic mutations in the epithelial mucosa and the inflammatory and stromal microenvironment ${ }^{16,17}$. AOM is a metabolite of the carcinogen 1,2-dimethylhydrazine (DMH) that does not directly result in DNA mutations. Instead, AOM is hydrolyzed to methylazoxymethanol (MAM) by the cytochrome isoform CYP2E1 in the liver, where MAM is conjugated with glucuronic acid and then transported to the intestine through bile secretions ${ }^{18}$. It is thought that the bacterial $\beta$-glucuronidase contributes to the degradation of MAM resulting in DNA alkylation and the accumulation of mutations in epithelial cells ${ }^{19}$. Most AOM-induced colonic tumors harbor missense mutations in the gene encoding $\beta$-catenin, rendering the protein resistant to proteasomal degradation, which results in aberrant activation of the canonical Wnt-signaling pathway ${ }^{20}$. When the activity of AOM is combined with the mucosal damage elicited by DSS, the ensuing wound healing response creates a microenvironment that is conducive to the growth and expansion of the mutagenized epithelium. In a variation of this model, repetitive administration of AOM alone over a period of several weeks can be used to model sporadic colorectal cancer, in the absence of DSS-induced colitis ${ }^{10,17}$. These two complimentary models provide experimental settings to study CAC and sporadic CRC respectively, both of which are associated with a pro-inflammatory tumor microenvironment ${ }^{10}$ 
The use of serial endoscopy in mice was pioneered by Becker and colleagues ${ }^{21}$, and enables longitudinal monitoring of colitis and tumor progression. Here we provide three pre-clinical protocols based on DSS-induced mucosal damage and/or AOM-mediated tumor induction to reproducibly induce specific colonic pathologies. The first protocol describes inducing acute mucosal damage in response to DSS administration to elicit many of the histopathological features associated with IBD. The second protocol is based on three consecutive cycles of DSS administration to mimic the flares of inflammation commonly observed in IBD patients, and can be carried out in conjunction with AOM-induced mutations. The final protocol is based on AOM-induced sporadic epithelial mutations. For each of these protocols, we expand on the relevant standard procedures to include prophylactic and therapeutic intervention methods that we have developed to monitor the efficacy of new drugs.

\section{Protocol}

The Walter and Eliza Hall Institute of Medical Research animal ethics committee approved each of the procedures described in these protocols

\section{Preparation of Experimental Mice}

1. Compose experimental cohorts of at least 4 gender matched 6-8 week old C57BL/6 mice (M. musculus) that are bred and housed in the same specific pathogen free (SPF) animal facility/room and provided with autoclaved food and water. The use of female mice will permit cohousing of mice from different lines, limit box numbers, and reduce cage-to-cage variation.

2. Ensure that mice are at least 6 weeks of age to enable endoscopy procedures using the $3.0 \mathrm{~mm}$ diameter endoscopy sheath.

3. Use mice of the same genetic background, and where possible, from cages stored on the same rack to reduce variations associated with the intestinal microflora. Variations in microflora between animal facilities should be taken into consideration when determining the appropriate control mice and genotypes for each experiment ${ }^{22}$

4. Mark mice by ear/toe clippings, tattoos, or similar to allow for easy identification.

5. Weigh mice on Day 0, to determine baseline experimental weights.

6. Perform endoscopy (see Section 5) of mice on Day 0 to record baseline colonic phenotypes.

\section{Pre-clinical Trial in an Epithelial Damage and Acute Colitis Model}

1. Prepare a solution of the therapeutic agent to be tested. For therapeutics delivered by oral gavage (p.o.), prepare solutions to a maximum volume of $100 \mu \mathrm{l}$. For therapeutics delivered by intraperitoneal (i.p.) injection, prepare solutions to a maximum volume of $200 \mu \mathrm{l}$. NOTE: Repeated i.p. or p.o. drug administration over prolonged periods of time can result in altered mouse behavior. Alternate the i.p. injection site to avoid prolonged discomfort. For p.o. administration, provide the mice with autoclaved sunflower seeds as a 'treat' to minimize the adverse association with the gavage procedure.

2. On Day 1, administer the therapeutic of interest (prepared in step 2.1) and appropriate vehicle controls. In the example provided (Figure 6A), $5 \mu \mathrm{g}$ of recombinant human (rh) ilnterleukin (IL)-11 protein was dissolved in $200 \mu \mathrm{l}$ phosphate buffered saline (PBS) and administered i.p.

3. Determine the timing and frequency of the administrations, which will be dependent on the pharmacokinetic profile established for the therapeutic reagent that is being tested. Figure 1 outlines prophylactic treatment using twice daily i.p. injections over the course of the experiment.

4. Monitor, including stool consistency and the presence of blood, and weigh each mouse daily. On the days of treatment, weighing should coincide with administration of the therapeutic drug to minimize stress to the mice caused by repeated handling.

5. On Day 3, prepare $2.5 \%(2.5 \mathrm{~g} / 100 \mathrm{ml})$ DSS solution in the drinking water routinely provided to the mice by the animal facility. Approximately $5 \mathrm{ml} / \mathrm{mouse} /$ day of DSS solution is required for the experiment. The consumption of the DSS water may change if the ambient temperature of the animal facility fluctuates.

NOTE: DSS is an irritant and should be handled in accordance with MSDS instructions.

6. Provide fresh DSS to the mice ad libitum in clean water bottles for 5 continuous days. On the evening of Day 5 , provide normal drinking water in addition to mashed food pellets and a protein supplement provided in small petri dishes (100 $\mathrm{g}$ food pellets $/ 10 \mathrm{~g}$ protein shake/10 ml water). This prevents dehydration and assists with minimizing severe weight-loss.

7. Euthanize mice by $\mathrm{CO}_{2}$ intoxication and collect tissues for subsequent histological analysis on the morning of Day 8 , or when the mice experience $\geq 15 \%$ weight-loss for more than three consecutive days, whichever occurs first.

NOTE: Adjust the DSS dose between 1-4\% (w/v) depending on the microflora of the animal facility and the batch of DSS. Carry out, routine batch testing to establish the appropriate DSS dosage and circumvent batch-to-batch variation. The appropriate dosage should be based on weight-loss (not greater than $15 \%$ of original weight) and confirmed colitis histopathology.

\section{Pre-clinical Trial in a Chronic Colitis or Colitis-associated Cancer Model}

1. For the chronic colitis model begin from step 3.7 .

2. For the colitis-associated cancer (CAC) model, on Day 1, inject each mouse with $10 \mathrm{mg} / \mathrm{kg}$ (w/w) Azoxymethane (AOM, $250 \mu \mathrm{l}$, i.p.). NOTE: AOM is a carcinogen and should be handled in accordance with MSDS instructions.

3. Stock solutions of $A O M$ are prepared as $10 \mathrm{mg} / \mathrm{ml}$ aliquots and stored at $-20^{\circ} \mathrm{C}$. On the day of injection, the AOM stock solution is thawed and diluted to $1 \mathrm{mg} / \mathrm{ml}$ in PBS. Repeated freezing and thawing of the AOM stock solution should be avoided.

4. The AOM dose may be adjusted between $8-12 \mathrm{mg} / \mathrm{kg}$. Routine batch testing is required to establish the appropriate AOM dosage in order to circumvent toxicity to mice due to batch-to-batch variation.

5. During Days 1-7 following AOM administration weights will be stable and for this reason weight monitoring can be omitted during Days 1-7 to avoid handling animals that will excrete cytotoxic metabolites in their feces.

6. On Day 7 all bedding needs to be changed following cytotoxic procedures. Once the bedding change is complete, the animals no longer require cytotoxic handling procedures, as they will no longer be excreting cytotoxic metabolites.

7. On Day 8, prepare $2.5 \%(2.5 \mathrm{~g} / 100 \mathrm{ml}$ ) DSS solution (as described in step 2.5$)$ and provide to the mice ad libitum. 
8. Weigh mice daily throughout the experiment and monitor for signs of illness, including ruffled fur, hunching, bloody stools and reduced movement.

9. On Day 13, remove the DSS solution and provide the mice with normal drinking water until Day 28. Days 8-28 constitute one 'cycle' of the chronic colitis/CAC protocol.

10. On Days 13-28, when the mice are given normal drinking water, provide the mice with mashed food pellets and a protein supplement provided in small petri dishes (100 g food pellets $/ 10 \mathrm{~g}$ protein shake $/ 10 \mathrm{ml}$ water). This prevents dehydration and assists with minimizing severe weight-loss.

NOTE: The protein supplements in mash should not be provided when the mice are receiving DSS-containing drinking water in order to ensure consistency in DSS consumption.

11. On Day 20 perform the second endoscopic examination to monitor individual mouse health (see Section 5).

12. On Day 29 repeat the DSS cycle (Cycle 2: $2.5 \%$ (w/v) DSS provided during Days 29-33 and normal drinking water during Days $34-50$ ).

13. On Day 40 perform the third endoscopic examination to monitor individual mouse health and determine tumor burden (see Section 5 ).

14. If no tumors are visible by endoscopy on Day 40, begin a third DSS cycle on Days 50-72 (Cycle 3: 2.5\% (w/v) DSS provided during Days 50-55 and normal drinking water during Days 56-72). Generally, tumors are visible by Day 40 and therefore the third cycle of DSS can be omitted.

15. Once tumors become visible by endoscopic examination, assign each mouse an endoscopic tumor score (see Section 5) and assign individual mice to cohorts with similar baseline tumor burdens.

16. On Day 42 (or when tumors are visible), administer the therapeutic compound or relevant vehicle control (See Section 2.2). The timing of the doses will be dependent on the pharmacokinetic profile of the therapeutic. An example of an intervention treatment injected i.p. three times weekly in mice with established tumors is provided (Figure 2).

17. Perform endoscopic exams once per week over the duration of the therapeutic treatment to monitor tumor burden. Generally, 4 weeks of a therapeutic treatment is sufficient to observe an objective treatment response. Following cessation of the treatment, a cohort of mice can also be monitored by endoscopy for tumor relapse.

18. Euthanize mice by $\mathrm{CO}_{2}$ intoxication and collect tissues for biochemical and/or histological analysis on the morning of Day 72 or when mice experience $\geq 15 \%$ weight-loss for more than three consecutive days, whichever occurs first.

\section{Pre-clinical Trial in a Sporadic Colorectal Cancer Model}

1. On Day 1, inject each mouse i.p. with $10 \mathrm{mg} / \mathrm{kg} \mathrm{AOM} \mathrm{(see} \mathrm{step} \mathrm{3.2).} \mathrm{The} \mathrm{AOM} \mathrm{dose} \mathrm{may} \mathrm{be} \mathrm{adjusted} \mathrm{between} \mathrm{8-12} \mathrm{mg/kg.} \mathrm{Routine} \mathrm{batch}$ testing is required to establish the appropriate AOM dosage in order to circumvent toxicity to mice due to batch-to-batch variation.

2. Repeat the injection on the first day of each week for the following 5 weeks (Figure 3 ). The mice should be handled according to cytotoxic safety procedures for the entire 6 weeks.

3. Weigh each mouse on the day of AOM injection to minimize distress to the mice caused by repeated handling NOTE: AOM is a carcinogen and should be handled in accordance with MSDS instructions.

4. All animals should be handled according to cytotoxic safety procedures for the 6 weeks of AOM i.p. injections required for this protocol.

5. During week 8 perform endoscopic examination to monitor for emerging tumors (see Section 5). Endoscopic examination should be performed every two weeks until tumors become visible. Generally, wild-type C57BI/6 animals begin to develop tumors by approximately 40 weeks after the initial administration of AOM.

6. Once tumors become visible by endoscopic examination, assign each mouse an endoscopic tumor score (see Section 5) and assign individual mice to a cohort with similar tumor burdens prior to the commencement of therapeutic treatment.

7. During week 40 (or when tumors are first visible), administer the therapeutic compound or the relevant vehicle control.

8. The timing of the doses will be dependent on the pharmacokinetic profile of the therapeutic (see step 2.1). An example of an intervention treatment injected i.p. 3 times weekly in mice with established tumors is provided (Figure 3 ).

9. Perform endoscopic examination weekly for the duration of the therapeutic treatment periods to monitor tumor burden. Generally, 4 weeks of a therapeutic treatment is sufficient for an objective treatment response. Following treatment cessation, a cohort of mice can also be monitored by endoscopy for tumor relapse.

10. Euthanize mice by $\mathrm{CO}_{2}$ intoxication and collect tissues for biochemical and/or histological analysis of the colon and tumors during week 45 , or following 4 weeks of therapy.

\section{Endoscopic Examinations}

1. The endoscopy equipment should be assembled according to standard procedures ${ }^{16}$

2. Sterilize and clean the endoscope probe with $70 \%$ ethanol or an anti-bacterial lubricant.

3. Videos can be recorded using a laptop or desktop computer and standard media software, such as iMovie. A standard computer monitor, rather than a medical grade monitor, is sufficient to visualize the colon during the endoscopy procedure (Figure 4).

4. Anesthetize groups of 5-6 mice simultaneously in a chamber with $3 \%$ isoflurane in $100 \% \mathrm{O}_{2}$ at a rate of $0.2-0.4 \mathrm{~L} / \mathrm{min}$. Once the mice are anesthetized, which is confirmed by toe pinch; the isoflurane levels should be altered to $0.5-2 \%$ for maintenance.

5. Remove an individual mouse from the chamber, and place it ventral side up with its head secured in a nose-cone. Full anesthesia should be monitored and the hind legs should be adjusted so that they are stretched out behind the mouse.

6. Place a vet ointment on the eyes of the mice to prevent dryness.

7. Hold the tail of the mouse where it meets the lower spine to clearly reveal the anus. Insert the rigid endoscope sheath into a beaker of water and adjust the airflow to allow the release of one small bubble at a time.

8. The airflow will permit the colon to inflate. If the airflow is too strong, air will be pumped into the stomach of the mouse. No additional lubrication is required.

9. Carefully insert the rigid endoscope sheath into the rectum. Generally, the endoscope can be inserted up to $3 \mathrm{~cm}$, at which point the colon in mice curves and is not accessible to the rigid endoscope.

10. A common difficulty during endoscopy procedures is blockage of access to the lumen of the colon due to fecal matter. Avoid this by gently massaging of the belly of mice prior to the procedure to encourage defecation, or carefully maneuvering the endoscope around the fecal 
matter during the procedure. Peristalsis can also occur, during which the endoscope should be held in position until the colon musculature relaxes.

11. Initiate video recording at any stage of the endoscopy procedure. Disease scoring can be recorded by an experienced assistant during the procedure, or at a later time point from the video files.

12. Following the endoscopic exam, return the mice to their cage and monitor them during recovery from anesthesia. Animals are generally awake and mobile within 2 min following removal from the isoflurane administering nose cone.

13. Ensure that these procedures are performed by an experienced scientist. Each endoscopy procedure will take approximately 2 min per mouse.

\section{Disease Scoring}

1. To monitor colitis, score videos for the Murine Endoscopic Index of Colitis Severity (MEICS) ${ }^{16}$, which documents changes in (i) the thickness of the colon wall indicated by transparency, (ii) stool consistency, (iii) blood vessel integrity and presence, (iv) ulcers and areas of regeneration that present in a granular fashion, (v) bleeding indicated by fibrin deposits (Figure 5a).

2. To monitor tumor burden, score videos for tumor incidence and size. Tumor size is determined by the diameter of the colonic lumen occupied by the tumor ${ }^{16}$ (Figure 5b). It is common to use both colitis and tumor scoring parameters for a single mouse. Using endoscopy, individual tumors and the health of the colonic mucosa can be monitored over time, providing a read-out for the success of new therapeutics in a single animal.

3. (Optional) Still images can be extracted from the videos for generation of representative figures (Figure 6).

\section{Representative Results}

Weight-loss is used as a standard parameter to monitor for disease associated with colitis, which is routinely used to monitor the overall health of mice. Animals generally maintain their weight while DSS-containing water is administered, and only start to loose weight when they are returned to normal drinking water. Acceptable weight-loss parameters should be established in accordance with the institution's animal ethics committee. In order to prevent the dehydration associated with prolonged diarrhea, use the routine provision of mash (food pellets mashed and mixed with drinking water) supplemented with a protein shake in addition to normal drinking water.

As an alternative anesthesia, ketamine/xylazine or similar agents can be used, if isoflurane equipment is not available. Due to the rigid nature of the endoscope, these procedures only allow for visualization of the most distal $3 \mathrm{~cm}$ of the mouse colon. Newer endoscopes with additional capabilities (including flexibility and fluorescence) are available depending on the needs of the experiment. However, since the deleterious effects of DSS are primarily limited to the distal colon of mice, with milder pathology observed in the middle colon, the rigid endoscope does not hinder monitoring of the mucosal health of individual mice. Although we describe a protocol for prophylactic treatment of acute DSS-induced colitis, this protocol can easily be modified to test intervention treatment strategies. The efficacy of a drug designed to alleviate epithelial damage and colitis can be monitored longitudinally in individual mice and quantified based on the scoring parameters described (Figure 5). This is advantageous over traditional experimental designs, which require culling of mice at specific time points during the experimental protocol, and does not permit characterization of the disease response to a treatment over time.

Clinical studies in humans have highlighted considerable variability in how different tumors in an individual patient respond to treatments. The procedures that are described here provide a means to monitor overall tumor burden, as well as the treatment response of individual tumors over the course of an experiment. It is important to consider that the intervention protocols that are outlined for the cancer models do not take into account the effects of a therapy on tumor initiation. Prophylactic protocols, with the treatment provided prior to when tumors become visible by endoscopy, are required to obtain this information. The protocols outlined here provide information on therapeutic effects on the on the progression (measured by tumor size) of individual tumors. Tumor regression may also be indicated by a reduction in the number of visible tumors.

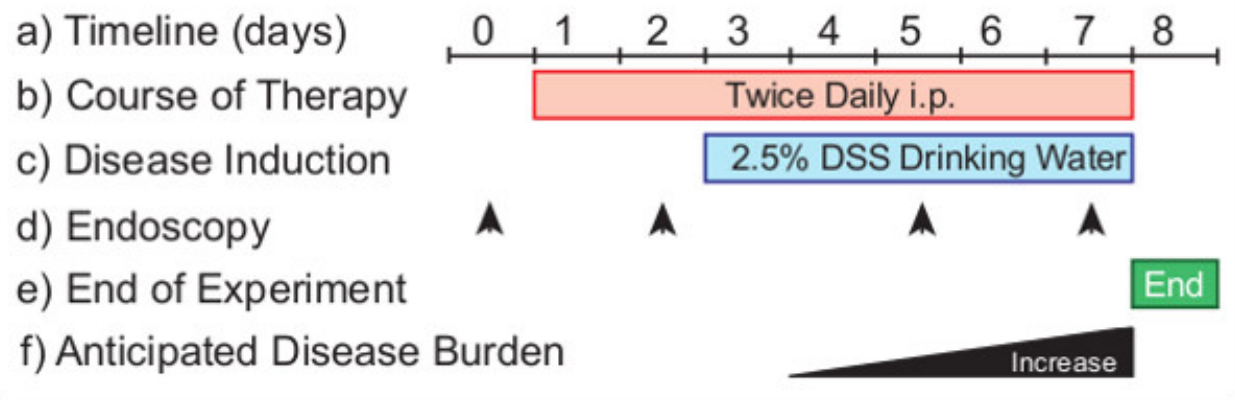

Figure 1: Monitoring the efficacy of a prophylactic treatment therapy in a model of acute colonic damage. The experimental protocol (a) requires 8 days from start to completion. Therapeutics are administered (b) from Day 1 for prophylactic treatments. DSS is provided in the drinking water (c) from Day 3 of the experimental protocol. Endoscopy is performed (d) to monitor the disease progression in the animals. Suggested timepoints include Day 0 (untreated) and Day 2 (health monitoring), Day 5 and 8 (to determine disease burden). The experiment is terminated (e) the morning of Day 8 . In a wild-type mouse (f) the progression of disease increases over time. 


a) Timeline (days)
b) Course of Therapy
c) Disease Induction
d) Endoscopy
e) End of Experiment
f) Anticipated Disease Burden

Figure 2: Monitoring the efficacy of intervention therapy in a model of colitis-associated cancer. The experimental protocol (a) requires 72 days from start to completion. Therapeutics (b) are administered to mice with established tumors from Day 46 for intervention treatments. AOM (c) is injected on Day 1, and DSS is provided in the drinking water over the course of three cycles of the experimental protocol, beginning on Day 8. Endoscopy (d) is performed to monitor the disease progression in the animals. Suggested timepoints include Day 0 (untreated), Day 20 (health monitoring), and Day 40 (to group animals according to tumor burden). Endoscopy is performed weekly over the course of the therapeutic treatment to monitor disease outcomes. The experiment (e) is terminated the morning of Day 72 . In a wild-type mouse (f) tumor burden increases from Day 40 onwards.

a) Timeline (weeks) 1
b) Course of Therapy
c) Disease Induction
d) Endoscopy
e) End of Experiment
f) Anticipated Disease Burden

Figure 3: Monitoring the efficacy of intervention therapy in a model of spontaneous colorectal cancer. The experimental protocol (a) requires $>50$ weeks from start to completion. Therapeutics (b) are administered to mice with established tumors for intervention treatments. AOM (c) is injected on Day 1, and weekly thereafter for 6 consecutive injections over the course of the experimental protocol. Endoscopy (d) is performed to monitor the disease progression in the animals. Suggested timepoints include Day 0 (untreated) and Week 8 (tumor monitoring) and biweekly there after (to establish tumor burden). Endoscopy is performed weekly over the course of treatment to monitor therapeutic outcomes. The experiment (e) is terminated in Week 50. In a wild-type mouse (f) tumor burden increases from Week 40 onwards.

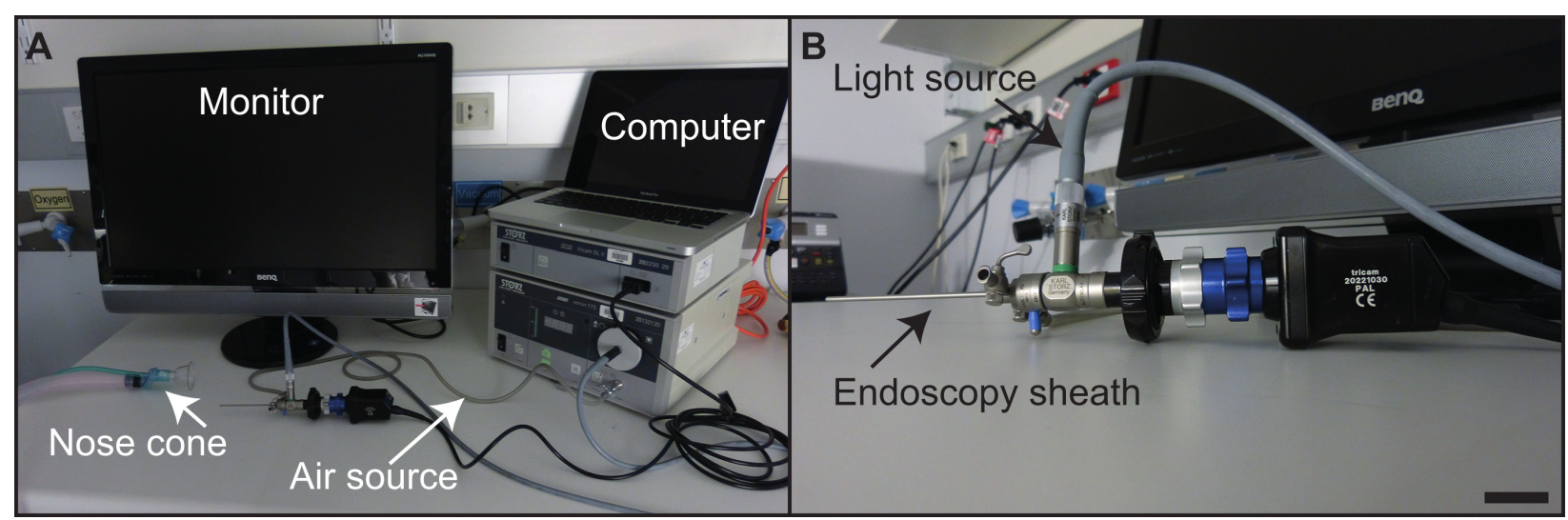

Figure 4: Equipment set-up. The experimental set-up (a) for the endoscopic unit, with individual pieces of equipment indicated. The rigid endoscope (b), with individual components indicated. Scale bar $=2.5 \mathrm{~cm}$. Please click here to view a larger version of this figure. 
a) Colitis Scoring

\begin{tabular}{|c|c|c|c|c|c|}
\hline & & & & & \multirow[b]{2}{*}{ Total } \\
\hline & 0 & 1 & 2 & 3 & \\
\hline Thickening of the colon & Transparent & Mild & Severe & Non-transparent & $0-3$ \\
\hline Stool consistency & Normal & Soft & Loose & Runny & $0-3$ \\
\hline Granularity & Smooth & Mild & Moderate & Cobblestones & $0-3$ \\
\hline Changes in vasculature & None & Mild & Moderate & Severe & $0-3$ \\
\hline Fibrin & None & Mild & Moderate & Severe & $0-3$ \\
\hline
\end{tabular}

b) Individual Tumor Scoring

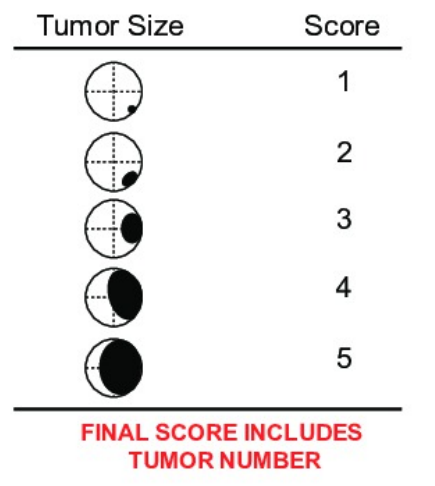

Figure 5: Scoring disease parameters by endoscopy. An outline (a) of the Murine Endoscopic Index of Colitis Severity (MEICS). An outline (b) of the individual tumor scoring parameters. Please click here to view a larger version of this figure.
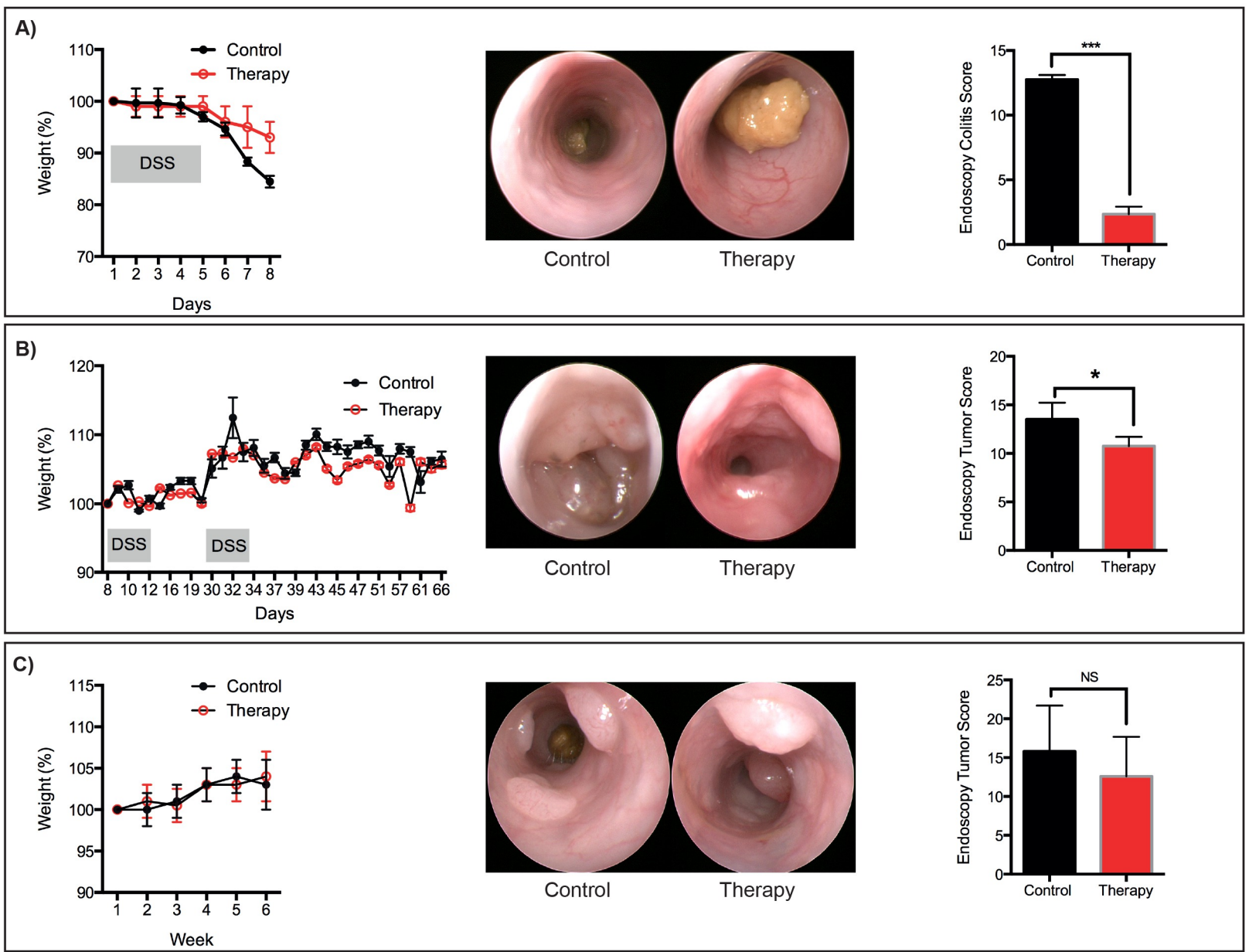

Figure 6: Representative therapeutic treatments. Representative weight-loss, endoscopic images and scores for: (a) Acute DSS-induced mucosal damage. (b) Tumors that developed following the AOM/DSS protocol. (c) Tumors that developed following the sequential AOM protocol. $\mathrm{N}=3$ mice per group. ${ }^{*} \mathrm{P}<0.05,{ }^{* *} \mathrm{P}<0.001$ (Student's T-test). Please click here to view a larger version of this figure. 


\section{Discussion}

The three protocols that are described outline methods of reliable and reproducible induction of colonic disease pathology in mice. When combined with routine endoscopic monitoring and the intervention strategies outlined here, these protocols will provide powerful pre-clinical insight into the efficacy of therapeutics. Our laboratories routinely use all of these protocols to monitor the success of novel therapeutics ${ }^{10,23,24}$.

There are a number of considerations when choosing a pre-clinical animal model to test new therapeutics. These include relevance of the model to the human disease, and the contribution of the tumor microenvironment to the proposed action of the therapeutic target. Here we provide three protocols for therapeutic intervention in established intestinal disease models. These models are reproducible and the delivery of reagents to induce disease is easy to manage. Importantly, the models are highly relevant to multiple facets and stages of colitis onset, and of tumor initiation and progression. Researchers should take into consideration the genetic background of the mouse strains used when designing experiments, as the susceptibility to disease induced by DSS and/or AOM can vary considerably ${ }^{25}$. In addition, different microbial communities may have different metabolic capacities in the context of AOM, which is metabolized by bacteria. We caution against using different cohorts of mice that were born into different animal facilities (including commercial vendors) in a single experiment. Similarly, the different microflora in mice used from different facilities may elicit different host responses to DSS-induced epithelial barrier damage ${ }^{11}$. Moreover, the appropriate analysis of tissue (for example RNA purification) should also be considered, since the ability of DSS to inhibit reverse transcriptase will impact on subsequent molecular analysis ${ }^{26,27}$.

Mouse endoscopy is a cutting edge technique to repeatedly monitor disease onset and progression in an individual mouse. The ability to record videos and extract still images permits easy monitoring of multiple disease parameters and tumors. In addition to improving animal welfare, endoscopic monitoring also reduces the need for multiple cohorts of experimental mice, which traditionally were culled at different time-points to track disease outcome. The MEICS scoring system is not a substitute for histopathological analysis, but provides an alternative means to monitor animal health and mucosal damage in live mice. Mouse endoscopy is a specialized laboratory technique, and all procedures should be performed by trained personnel to ensure appropriate manipulation and handling of the mice, as well as to provide consistent quality in the images used for disease scoring. In the hands of qualified personal, we have found that endoscopy induces little or no damage to the tumors that would cause intra-tumoral bleeding. For the therapeutic protocols outlined, we consider endoscopy highly advantageous, since it provides a way to determine the initial tumor burden, and allows us to group cohorts of animals with similar tumor burdens prior to the administration of a therapeutic drug. Sequential monitoring of the mice enables researchers to determine the efficacy of novel therapies early on, with the option of terminating unsuccessful experiments in a timely manner.

As our understanding of inflammatory bowel disease and colorectal cancers advance, new targets for therapy will be identified. Appropriate animal models will be integral to ensuring that the most promising new therapies are moved towards clinical trials.

\section{Disclosures}

The authors have nothing to disclose.

\section{Acknowledgements}

We would like to thank CSL Ltd. for supporting the purchase of the endoscopy equipment. The research in the laboratory of ME is supported by the Ludwig Institute for Cancer Research, and the laboratories of TP and ME are supported by the Victorian State Government Operational Infrastructure Support and the National Health and Medical Research Council of Australia. ME is an NHMRC Senior Research Fellow.

\section{References}

1. Tenesa, A., Dunlop, M. G. New insights into the aetiology of colorectal cancer from genome-wide association studies. Nat Rev Genet. 10, 353-358 (2009).

2. Rustgi, A. K. The genetics of hereditary colon cancer. Genes Dev. 21, 2525-2538 (2007).

3. Rutter, M., et al. Severity of inflammation is a risk factor for colorectal neoplasia in ulcerative colitis. Gastroenterology. 126, 451-459 (2004).

4. Eaden, J. A., Abrams, K. R., Mayberry, J. F. The risk of colorectal cancer in ulcerative colitis: a meta-analysis. Gut. 48, 526-535 (2001).

5. Lakatos, P. L., Lakatos, L. Risk for colorectal cancer in ulcerative colitis: changes, causes and management strategies. World $J$ Gastroenterol. 14, 3937-3947 (2008).

6. Xiang, B., Snook, A. E., Magee, M. S., Waldman, S. A. Colorectal cancer immunotherapy. Discov Med. 15, 301-308 (2013).

7. Feng, Q. Y., et al. Anti-EGFR and anti-VEGF agents: Important targeted therapies of colorectal liver metastases. World J Gastroenterol. 20, 4263-4275 (2014).

8. Dinarello, C. A. Anti-inflammatory Agents: Present and Future. Cell. 140, 935-950 (2010)

9. Grivennikov, S. I., et al. Adenoma-linked barrier defects and microbial products drive IL-23/IL-17-mediated tumour growth. Nature. 491, 254-258 (2012).

10. Putoczki, T., Thiem, S., Loving, A., Busuttil, R. A., Wilson, N. A., Ziegler, P., Nguyen, P., Preaudet, A., Farid, R., Edwards, K., Boglev, Y., Luwor, R. B., Jarnicki, A. J., Horst, D., Boussioutas, A., Heath, J., Sieber, O., Nash, A., Greten, F., McKenzie, B. S., Ernst, M. Interleukin-11 is the dominant IL-6 family cytokine during gastrointestinal tumorigenesis and can be targeted therapeutically. Cancer Cell. 24, 257-271 (2013).

11. Perse, M., Cerar, A. Dextran sodium sulphate colitis mouse model: traps and tricks. J Biomed Biotechnol. 2012, 718617 (2012).

12. Rose, W. A. 2nd, Sakamoto, K., Leifer, C. A. Multifunctional role of dextran sulfate sodium for in vivo modeling of intestinal diseases. BMC Immunol. 13, 41 (2012).

13. Laroui, H., et al. Dextran sodium sulfate (DSS) induces colitis in mice by forming nano-lipocomplexes with medium-chain-length fatty acids in the colon. PLoS One. 7, e32084 (2012). 
14. Miyazawa, F., Olijnyk, O. R., Tilley, C. J., Tamaoki, T. Interactions between dextran sulfate and Escherichia coli ribosomes. Biochim Biophys Acta. 145, 96-104 (1967).

15. Peterson, L. W., Artis, D. Intestinal epithelial cells: regulators of barrier function and immune homeostasis. Nat Rev Immunol. 14, 141-153 (2014).

16. Neufert, C., Becker, C., Neurath, M. F. An inducible mouse model of colon carcinogenesis for the analysis of sporadic and inflammationdriven tumor progression. Nat Protoc. 2, 1998-2004 (2007).

17. Schwitalla, S., et al. Loss of p53 in Enterocytes Generates an Inflammatory Microenvironment Enabling Invasion and Lymph Node Metastasis of Carcinogen-Induced Colorectal Tumors. Cancer Cell. 23, 93-106 (2013).

18. Fiala, E. S. Investigations into the metabolism and mode of action of the colon carcinogens 1,2-dimethylhydrazine and azoxymethane. Cancer. 40, 2436-2445 (1977).

19. Chen, J., Huang, X. F. The signal pathways in azoxymethane-induced colon cancer and preventive implications. Cancer Biol Ther. 8, 1313-1317 (2009).

20. Bollrath, J., et al. gp130-mediated Stat3 activation in enterocytes regulates cell survival and cell-cycle progression during colitis-associated tumorigenesis. Cancer Cell. 15, 91-102 (2009).

21. Becker, C., Fantini, M. C., Neurath, M. F. High resolution colonoscopy in live mice. Nat Protoc. 1, 2900-2904 (2006).

22. Ivanov, I. I., et al. Induction of intestinal Th17 cells by segmented filamentous bacteria. Cell. 139, 485-498 (2009).

23. Thiem, S., et al. mTORC1 inhibition restricts inflammation-associated gastrointestinal tumorigenesis in mice. J Clin Invest. 123, 767-781 (2013).

24. Stuart, E., et al. Therapeutic inhibition of Jak activity inhibits progression of gastrointestinal tumors in mice. Mol Cancer Ther. 13, 468-474 (2014).

25. De Robertis, M., et al. The AOM/DSS murine model for the study of colon carcinogenesis: From pathways to diagnosis and therapy studies. $J$ Carcinog. 10, 9 (2011).

26. Viennois, E., Chen, F., Laroui, H., Baker, M. T., Merlin, D. Dextran sodium sulfate inhibits the activities of both polymerase and reverse transcriptase: lithium chloride purification, a rapid and efficient technique to purify RNA. BMC Res Notes. 6, 350 (2013).

27. Kerr, T. A., et al. Dextran sodium sulfate inhibition of real-time polymerase chain reaction amplification: a poly-A purification solution. Inflamm Bowel Dis. 18, 344-348 (2012). 